\title{
RIPChallenge: Um Jogo Sério para Auxílio ao Aprendizado do Protocolo RIP
}

\author{
Helcio Wagner da Silva
}

\author{
Departamento de Computação - Universidade Federal Rural do Semi-Árido (UFERSA) \\ Caixa Postal 59.625-900 - Mossoró - RN - Brasil \\ helciodufersa.edu.br
}

\begin{abstract}
The learning of dynamic routing protocols requires complementary approaches because of their complexity. One of such approaches is the use of serious games. This paper describes the development of RIPChallenge, a serious game specifically aimed to boost the learning of the RIP protocol. The game has as major points its easiness to play and the addressing of protocol's operating characteristics students report more dificult to learn about. It was validated using the EGameFlow methodology, which shown it helped students to get a better confidence about the understanding of those characteristics.
\end{abstract}

Resumo. Haja vista sua complexidade, o aprendizado de protocolos de roteamento dinâmico requer abordagens complementares às tradicionais. Uma destas abordagens é a utilização de jogos sérios. Este artigo descreve o desenvolvimento do RIPChallenge, um jogo sério destinado especificamente ao auxílio da aprendizagem do protocolo RIP. O jogo destaca-se por ser de fácil manuseio e ter seu foco nos aspectos do protocolo que possuem maior dificuldade de aprendizado por parte dos estudantes. Sua validação, realizada com o auxilio da metodologia EGameFlow, demonstrou que ele proporciona uma melhor confiança acerca da compreensão daqueles aspectos.

\section{Introdução}

A Internet é constituída, em última instância, por uma interconexão de diversas redes de computadores. Entre estas redes figuram equipamentos de comutação denominados roteadores. O papel de um roteador é receber pacotes por uma de suas portas e encaminhá-los por outra porta. A porta de saída é descoberta através de um processo conhecido como roteamento, e para isto o roteador conta com uma tabela de rotas. $\mathrm{O}$ preenchimento automático das tabelas de rotas dos roteadores é feito por protocolos de roteamento dinâmico. Tradicionalmente, o estudo acadêmico dos protocolos de roteamento dinâmico é muito superficial, tanto pelo fato de que cada um deles é repleto de detalhes quanto pelo fato de que muita atenção é dada à matemática que compõe os algoritmos nos quais eles são baseados. Esta superficialidade explica em parte a exigência de certificações em redes de computadores no tocante ao roteamento.

A gamificação é definida como a aplicação de elementos de jogos em cenários que não são propriamente jogos, como em estratégias de marketing voltadas aos consumidores, em ambientes corporativos para o engajamento de funcionários e, por último, em escolas e universidade com vistas a aumentar o desempenho dos estudantes [Kapp 2012]. Os jogos voltados à instrução, em oposição àqueles que têm como finalidade apenas o entretenimento, são denominados jogos sérios [Michael e Chen 2005]. Em [Hays 2005] é destacado que a efetividade dos jogos não pode ser 
generalizada, sua utilização deve ser baseada em uma análise detalhada das necessidades de aprendizagem e balanceada entre outras abordagens instrucionais. Além disso, aquele estudo conclui que suporte instrucional aumenta a sua efetividade. $\mathrm{O}$ estudo de [Vogel et al 2006] conclui que a utilização de jogos estimula a elevação dos níveis cognitivos, principalmente quando os aprendizes podem utilizá-los livremente isto é, sem a supervisão dos instrutores. Este artigo apresenta RIPChallenge, um jogo sério para auxilio ao aprendizado do RIP, um dos protocolos de roteamento dinâmico da Internet. Para tal, ele está estruturado da seguinte forma: a próxima seção aborda o funcionamento básico do RIP; a Seção 3 descreve algumas ferramentas utilizadas como complemento ao ensino dos protocolos de roteamento dinâmico; a Seção 4 descreve alguns trabalhos sobre jogos voltados ao ensino do roteamento dinâmico; a Seção 5 ilustra o desenvolvimento do jogo RIPChallenge; a Seção 6 descreve seu processo de validação; a Seção 7 provê as considerações finais acerca do trabalho realizado, relacionando-o com aqueles descritos na Seção 4 e apresentando futuras direções.

\section{Fundamentos do RIP}

O RIP (Routing Information Protocol) é um dos mais antigos protocolos de roteamento dinâmico. Sua versão inicial (RIPv1) é especificada em [Hedrick 1988], enquanto o RIPv2 é especificado em [Malkin 1998]. O RIP implementa o algoritmo de BellmanFord, que também é conhecido como vetor de distâncias. Ao invés de descrever a operação do protocolo pela formulação matemática do algoritmo, opta-se aqui por prover um exemplo prático, ilustrado pela Figura 1.

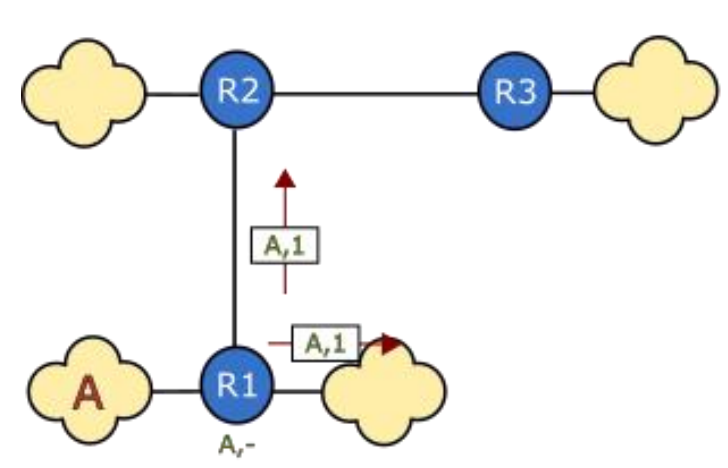

(a)

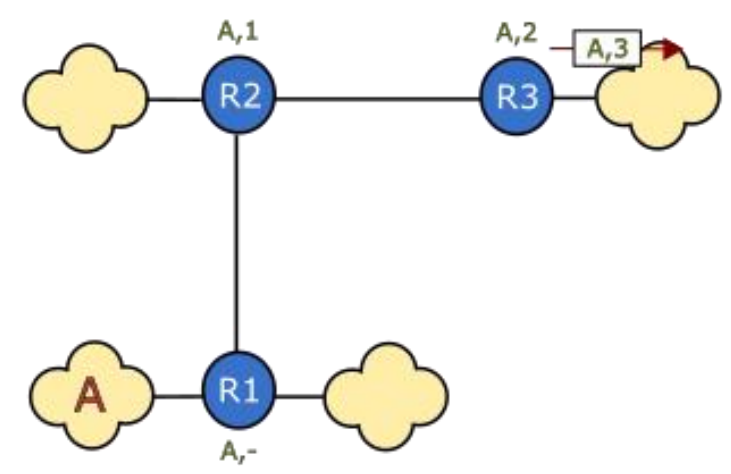

(c)

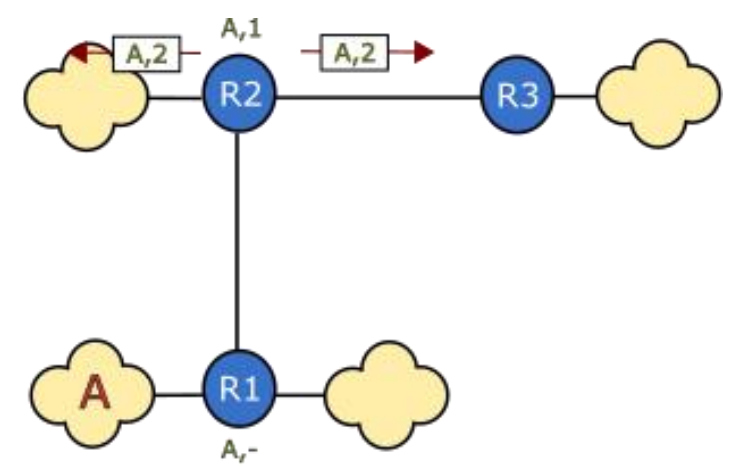

(b)

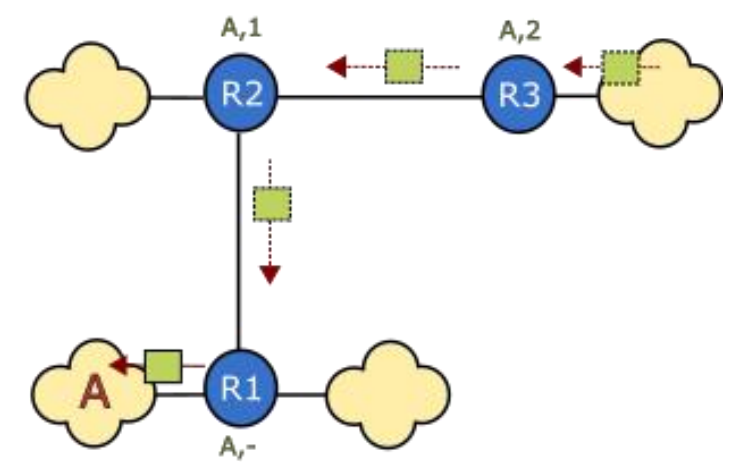

(d)

Figura 1. Exemplo de atualização de rota no RIP 
Em (a) surge uma rede $A$ diretamente conectada à interface esquerda do roteador R1. Este roteador adiciona uma entrada correspondente na sua tabela de rotas $(A,-)$ e anuncia uma rota para aquela rede pelas suas demais interfaces. A rota é definida como $(A, 1)$, sendo $A$ a identificação da rede e 1 o custo - para o RIP, o custo corresponde à quantidade de roteadores que devem ser percorridos para se atingir determinada rede.

Em (b), o roteador $\mathrm{R} 2$ recebe a rota de $\mathrm{R} 1$, adiciona uma entrada à sua tabela de rotas $(A, 1)$ e anuncia uma rota para a rede $A$ pelas suas demais interfaces; a rota é definida como $(A, 2)$. Em $(\mathrm{c}), \mathrm{R} 3$ recebe a rota de $\mathrm{R} 2$, adiciona uma entrada à sua tabela de rotas $(A, 2)$ e anuncia uma rota para a rede $A$ pela sua outra interface; a rota é definida como $(A, 3)$. Por fim, em $(d)$ um pacote destinado à rede $A$ é transmitido a partir da rede à direita de R3. O pacote é roteado por R3 para R2; assim que chega à R2, o pacote é roteado por R2 para R1; assim que chega à $\mathrm{R} 1$, o pacote é roteado por R1 para a rede $\mathrm{A}$. $\mathrm{O}$ fato de o pacote ter chegado ao seu destino só foi possível porque todos os roteadores possuíam uma rota para a rede $\mathrm{A}$ em suas respectivas tabelas de rota. Se ao menos um dos roteadores não possuísse uma entrada para a rede $\mathrm{A}$ em sua respectiva tabela de rotas, o pacote seria descartado assim que chegasse a ele.

Do exemplo exposto acima, algumas observações são importantes. A primeira delas diz respeito ao valor do custo inserido na tabela de rotas. Em particular, ele sempre corresponde ao valor do custo presente na rota recebida - no caso especial no qual a rede é diretamente conectada ao roteador, como é o caso da rede A com o roteador R1, o custo correspondente àquela rede é nulo (ou “-”). A segunda observação diz respeito ao valor do custo inserido na rota anunciada. Em particular, ele sempre corresponde ao valor do custo na tabela de rotas acrescido de 1 (um). Por fim, em topologias mais complexas que aquela ilustrada pela Figura 1, mais especificamente em topologias circulares, um roteador pode receber mais de uma rota para uma mesma rede. Em tais circunstâncias, ele escolhe sempre a rota que tiver o menor custo e a anuncia pelas suas demais interfaces.

\section{Ferramentas de Apoio ao Ensino do RIP}

Atualmente, o ensino dos protocolos de roteamento dinâmico nos cursos de redes de computadores nas instituições de ensino superior brasileiras conta com a ajuda de livros-texto consagrados, dentre eles [Tanenbaum e Wetherall 2011] e [Kurose e Ross 2013]. Todavia, tais referências se propõem a cobrir uma vasta gama de protocolos, e em alguns casos não fornecem uma cobertura suficientemente clara acerca de um determinado protocolo. Com relação ao RIP, por exemplo, elas dão ênfase ao formalismo matemático do algoritmo Bellman-Ford, e não às mensagens trocadas pelo protocolo e seus efeitos nas tabelas de rotas dos roteadores.

Há um certo tempo, os livros-texto são complementados, em alguns cursos, por ferramentas computacionais. A mais popular delas é o Packet Tracer, uma ferramenta de simulação criada pela Cisco Systems que oferece aos usuários uma interface do tipo drag and drop permitindo a criação de redes simuladas compostas por computadores, switches e roteadores, dentre outros. Esta ferramenta conta com dois modos: o modo de tempo real e o modo de simulação. No modo de simulação, o envio de mensagens pode ser controlado pelo usuário, e os campos de cada mensagem podem ser visualizados detalhadamente. Com versões para os sistemas operacionais Windows, Linux, Android e iOS, o Packet Tracer é originalmente destinado aos estudantes da Cisco Academy, a plataforma de ensino da Cisco, para obtenção de seus certificados CCNA (Cisco 
Certified Network Associate). Porém, qualquer pessoa pode obter a ferramenta a partir de [Cisco Systems 2019] mediante criação de uma conta.

Outra ferramenta muito popular é o GNS3 (Graphical Network Simulator 3), que também conta com uma interface drag and drop. Ela difere do Packet Tracer pelo fato de utilizar emulação, executando imagens de equipamentos reais através do recurso de virtualização. Isto possibilita a criação de cenários muito mais realistas que o Packet Tracer. Além disso, o GNS3 permite a utilização de equipamentos de diversos fabricantes, diferentemente da ferramenta da Cisco. Porém as imagens dos equipamentos devem ser obtidas separadamente, e sua emulação consome muito mais recursos computacionais que uma topologia simulada no Packet Tracer. Outra desvantagem do GNS3 em relação ao Packet Tracer é que, de forma geral, o envio das mensagens não é controlado pelo usuário, e seus campos só podem ser visualizados a partir de uma ferramenta externa de captura de pacotes (o wireshark). Com versões para os sistemas operacionais Windows, OS X Mavericks e Linux, o GNS3 pode ser obtido a partir de [GNS3 Technologies Inc. 2019] também mediante criação de uma conta.

\section{Trabalhos Relacionados}

Há uma grande quantidade de trabalhos descrevendo o projeto e desenvolvimento de jogos educacionais relacionados a várias áreas do conhecimento. Com relação ao ensino do roteamento dinâmico, são destacados aqui três deles. Em [Graven et al 2009], o roteamento dinâmico é representado de forma abstrata por um MMORPG (Massively Multiplayer Online Role-Playing Game) que utiliza um labirinto ao longo do qual jogadores recebem de um NPC (Non Player Character) um objeto que deve ser entregue para outro NPC. Ao final de cinco entregas os jogadores recebem pedaços de ouro que, em conjunto, podem ser trocados por itens na loja do jogo. Vence o jogador que adquire e veste uma camiseta, um dos itens da loja. Os jogadores percorrem cegamente o labirinto, orientados apenas por placas de distância, e desenham o seu traçado em folhas de papel. A partir daí, eles calculam mentalmente os melhores caminhos até os NPCs. A despeito do seu caráter abstrato, o jogo foi avaliado como sendo benéfico ao ensino do roteamento.

Em [Arevalillo-Herráez et al 2012] é apresentado "Conquer the Net", um jogo baseado em turnos para dois jogadores e que tem como pano de fundo uma interconexão de redes abrangendo vários países. Trata-se, portanto, de um jogo que utiliza um nível de abstração menor do que o apresentado no parágrafo anterior. Cada jogador recebe um PC e tem que acessar um serviço de rede diferente a partir da configuração dos equipamentos ao longo do caminho. Na sua vez, o jogador realiza a configuração dos equipamentos por meio de procedimentos cuja quantidade é determinada pelo lançamento de um dado virtual. Vence quem acessar o seu serviço primeiro. "Conquer the Net" é um jogo desenvolvido em Java para ser jogado pelos dois jogadores utilizando um único computador.

Um jogo sobre o roteamento em MANETs (Mobile Ad hoc NETworks) é apresentado em [Lemcke et al 2015]. Denominado "RouteMe", o jogo divide os jogadores em dois grupos. O primeiro deles age como os nós da MANET, e se espalham sobre um ambiente aberto de um campus equipados com seus smartphones e tablets. A interface do jogo para este grupo permite a um nó o estabelecimento de conexões com nós vizinhos e o roteamento de mensagens que chegam àquele nó para um dos seus vizinhos. Além disso, a interface permite ao nó a coleta de objetos virtuais que aumentam seu raio de alcance ou a duração de sua bateria. $\mathrm{O}$ vencedor deste grupo é o 
nó que consegue rotear mais mensagens. O segundo grupo utiliza PCs e laptops em um ambiente fechado para transmitir mensagens entre os nós do primeiro grupo. $\mathrm{O}$ vencedor daquele grupo é aquele que conseguiu a maior quantidade de transmissões bem-sucedidas. Possuindo três níveis de dificuldade, nos quais o automatismo dos procedimentos diminui gradativamente, "RouteMe" foi positivamente avaliado pelos estudantes.

\section{O jogo desenvolvido}

Desenvolvido com o auxílio do motor de jogos Unity, RIPChalenge pode ser executado em várias plataformas, como PCs e smartphones, por exemplo. A Figura 2 ilustra a sua tela inicial, contendo um menu com três opções. Considerando que as regras do jogo são razoavelmente simples, optou-se por disponibilizar como primeira opção a opção jogar.

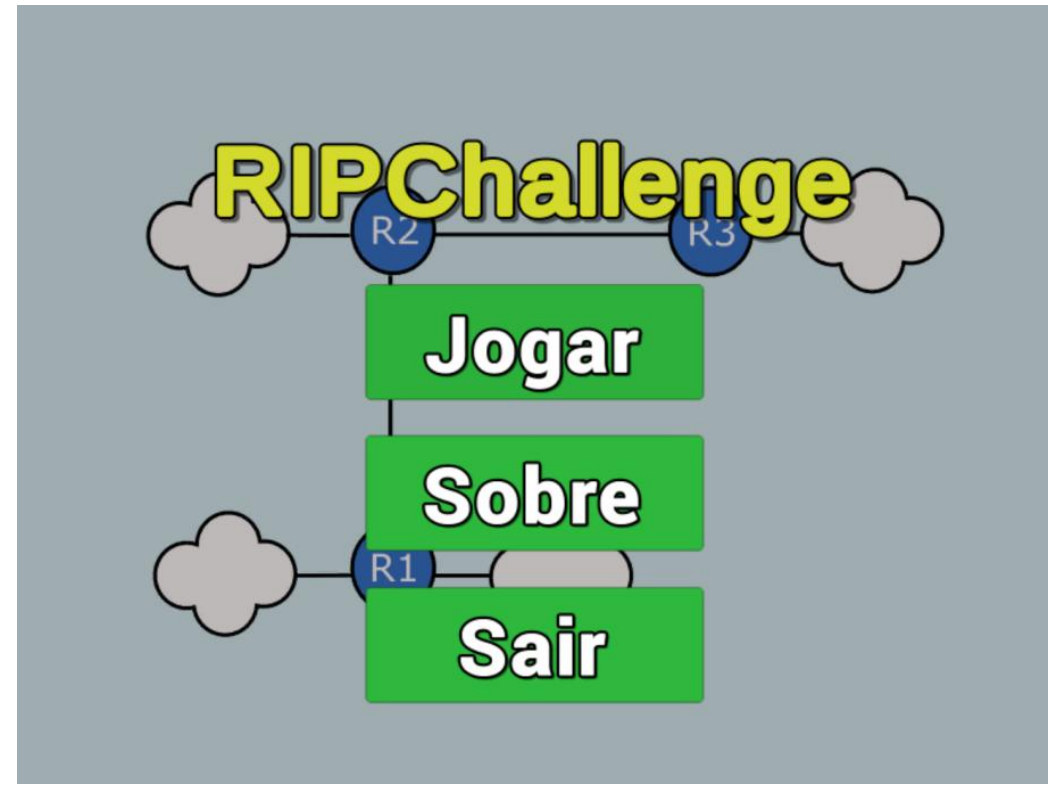

Figura 2: tela inicial de RIPChallenge.

O jogo possui quatro níveis de dificuldade, que vão do 1 ao 4. Eles diferenciam-se pela quantidade de tempo que o jogador dispõe para tomar decisões: o jogador dispõe de uma maior quantidade de tempo no nível 1 para a tomada de decisões, e essa quantidade diminui progressivamente até ele chegar ao nível 4. O RIPChallenge pode ser jogado de dois modos. No primeiro deles o nível de dificuldade aumenta automaticamente; isto é, o jogador inicia o jogo no nível 1 e o termina no nível 4. Neste modo, o tempo de duração em cada um dos níveis é de aproximadamente 40 segundos. No segundo modo, o jogador pode alterar o nível de dificuldade a seu bel prazer - este é um modo destinado ao treino, e nele a duração de cada nível é indefinida.

A Figura 4 ilustra uma captura de tela durante o gameplay de RIPChallenge, cujo objetivo é permitir que os três roteadores de uma interconexão similar àquela ilustrada pela Figura 1 roteiem a maior quantidade possível de pacotes. A dinâmica do jogo pode ser assim explicada: periodicamente, uma rede surge de maneira aleatória em uma das quatro nuvens da interconexão. O roteador mais próximo a ela tem sua cor alterada para vermelho e surgem uma rota na sua tabela de rotas e atualizações próximo às suas demais interfaces. Um assistente na forma de emoji instrui o jogador a clicar sobre o roteador. Isto faz com que as atualizações sejam disparadas. Quando uma delas chega 
até outro roteador diretamente conectado, este roteador tem sua cor mudada para vermelho e surgem uma rota na sua tabela de rotas e atualizações nas suas demais interfaces. O assistente surge próximo a ele, instruindo o jogador a clicar sobre ele para que as atualizações sejam disparadas.

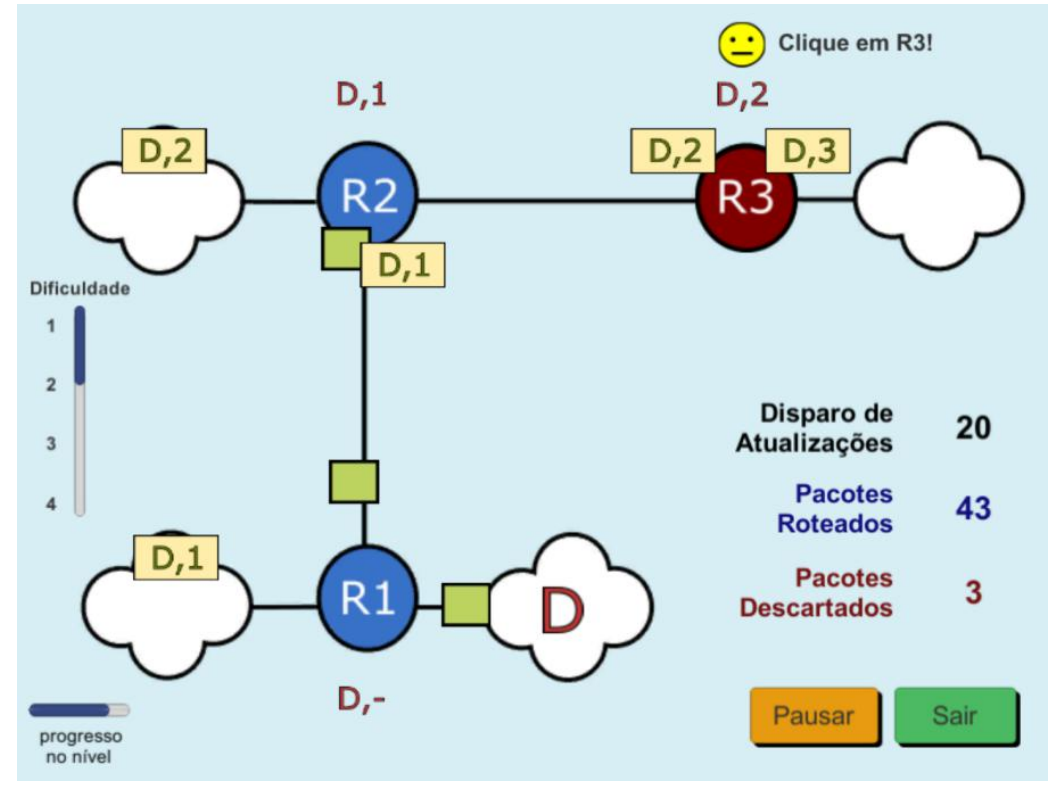

Figura 4: Captura de tela no andamento do jogo.

O jogador deve clicar sobre os roteadores assim que eles possuam atualizações para serem enviadas, de forma que cada roteador da interconexão possua uma rota para a rede. Isto deve ocorrer no menor tempo possível, porque um conjunto de três pacotes destinados àquela rede surgirá de maneira aleatória em outra nuvem da interconexão a atravessará um ou mais roteadores no caminho. Se o roteador possui uma rota para a rede, ele roteará o pacote; caso contrário, ele o descartará. Na Figura 4, que ilustra o jogo no seu modo padrão, a identificação do nível em que jogo está é fornecida por uma escala localizada à esquerda; um pouco abaixo dela está uma outra escala que fornece ao jogador uma indicação de quão próximo do final daquele nível ele está. À direita, localizam-se as indicações de quantas atualizações foram disparadas pelo jogador até então, quantos pacotes foram roteados com base naqueles disparos e quantos pacotes foram descartados pelo fato de que uma ou mais atualizações não foram disparadas ou foram disparadas tardiamente. Na captura ilustrada, a rede D surgiu na nuvem à direita de $\mathrm{R} 1$ e os pacotes destinados a ela foram enviados da nuvem à esquerda de R2. Todos eles chegarão ao seu destino, embora ainda reste ao jogador clicar em R3 para que ele envie a sua atualização $(D, 3)$ pela nuvem à sua direita. Até então, foram disparadas 20 atualizações, roteados 43 pacotes e descartados outros três. O jogo está no nível 2, próximo de evoluir para o nível 3. Quando o jogador passar pelos quatro níveis, o jogo será finalizado e uma tela de encerramento ilustrará o total de atualizações disparadas, de pacotes roteados e de pacotes descartados. Ao longo dos seu gameplay, o RIPChallenge conta com uma trilha sonora importada do asset Universe Sounds Free Pack. Além disso, os sons reproduzidos quando uma rede surge, um pacotes é roteado ou um pacote é descartado são fornecidos pela importação do asset Score and Times. Ambos assets possuem a maior reputação possível junto à comunidade Unity. Todos os elementos gráficos do jogo foram construídos com o auxílio do editor de gráficos vetoriais Inkscape, disponibilizado gratuitamente. 


\section{Validação}

Para validação do RIPChallenge, foi utilizado o método EGameFlow, definido em [Fu et al 2009]. Este método toma como base o método GameFlow, definido em [Sweetser e Wyeth 2006] para avaliação de jogos com base na Teoria do Fluxo [Csikszentmihalyi 1990]. O EGameFlow possui uma escala de oito dimensões, cada qual contendo um conjunto de itens que são analisados para a obtenção da nota da dimensão. A nota de cada item é fornecida por cada avaliador utilizando uma escala Likert de sete pontos, que vai de "discordo fortemente" (1) até "concordo fortemente" (7).

A Tabela 1 ilustra a escala do EGameFlow e as médias obtidas na avaliação do protótipo do RIPChallenge por alunos da disciplina "Interconexão de Redes", cuja ementa contempla os protocolos de roteamento dinâmico - dentre eles o RIP. A avaliação foi feita através de prática em sessões sem limite de tempo após aquele protocolo ter sido apresentado à turma de forma tradicional.

Tabela 1. Escala do EGameFlow com as médias obtidas na avaliação.

\begin{tabular}{|c|c|c|c|}
\hline Dimensão & Item & Conteúdo & Notas \\
\hline \multirow{9}{*}{ Concentração } & $\mathrm{C} 1$ & O jogo prende minha atenção? & 6,0 \\
\hline & $\mathrm{C} 2$ & Apresenta conteúdo que estimula minha atenção? & 6,0 \\
\hline & $\mathrm{C} 3$ & $\begin{array}{l}\text { A maioria das atividades está relacionada com a tarefa da } \\
\text { aprendizagem? }\end{array}$ & 6,0 \\
\hline & $\mathrm{C} 4$ & Nenhuma distração da tarefa é destacada? & 5,5 \\
\hline & $\mathrm{C} 5$ & No geral, consigo ficar concentrado no jogo? & 7,0 \\
\hline & C6 & Não sou distraído de tarefas nas quais deveria me concentrar? & 7,0 \\
\hline & $\mathrm{C} 7$ & Não sou sobrecarregado com tarefas que parecem sem importância? & 5,0 \\
\hline & $\mathrm{C} 8$ & A carga de trabalho do jogo é adequada? & 6,5 \\
\hline & & Média da dimensão & 6,1 \\
\hline \multirow{11}{*}{ Desafios } & $\mathrm{H} 1$ & Aproveito o jogo sem ficar entediado ou ansioso? & 6,0 \\
\hline & $\mathrm{H} 2$ & Dificuldade é adequada? & 6,0 \\
\hline & $\mathrm{H} 3$ & Existem "dicas" que ajudam na tarefa? & 5,5 \\
\hline & $\mathrm{H} 4$ & Apresenta suporte on-line que ajuda na tarefa? & 1,5 \\
\hline & H5 & Apresenta vídeo ou áudio que ajudam na tarefa? & 3,0 \\
\hline & H6 & Minhas habilidades aumentam conforme o jogo avança? & 6,5 \\
\hline & $\mathrm{H} 7$ & Sou motivado pela melhora das minhas habilidades? & 5,5 \\
\hline & $\mathrm{H} 8$ & Os desafios aumentam conforme minhas habilidades aumentam? & 6,5 \\
\hline & $\mathrm{H} 9$ & Apresenta novos desafios em um ritmo adequado? & 6,0 \\
\hline & $\mathrm{H} 10$ & $\begin{array}{l}\begin{array}{l}\text { Apresenta diferentes níveis de desafios que se adaptam aos } \\
\text { diferentes jogadores? }\end{array} \\
\end{array}$ & 6,5 \\
\hline & \multicolumn{2}{|r|}{ Média da dimensão } & 5,3 \\
\hline \multirow{10}{*}{ Autonomia } & A1 & Tenho sensação de controle do menu? & 5,5 \\
\hline & $\mathrm{A} 2$ & Tenho sensação de controle sobre funções e objetos? & 5,5 \\
\hline & A3 & $\begin{array}{l}\text { Tenho sensação de controle sobre as interações entre funções e } \\
\text { objetos? }\end{array}$ & 6,0 \\
\hline & A4 & Não é possível cometer erros que impedem o avanço do jogo? & 7,0 \\
\hline & A5 & Posso me recuperar de qualquer erro cometido? & 4,5 \\
\hline & A6 & Sinto que posso usar quaisquer estratégias? & 3,5 \\
\hline & A7 & Tenho sensação de controle e impacto sobre o jogo? & 6,5 \\
\hline & A8 & Sei o próximo passo no jogo? & 6,0 \\
\hline & A9 & Tenho sensação de controle sobre o jogo? & 6,5 \\
\hline & \multicolumn{2}{|r|}{ Média da dimensão } & 5,7 \\
\hline \multirow{4}{*}{$\begin{array}{l}\text { Clareza dos } \\
\text { Objetivos }\end{array}$} & G1 & Objetivos gerais apresentados no início do jogo? & 6,5 \\
\hline & G2 & Objetivos gerais apresentados claramente? & 6,5 \\
\hline & G3 & Objetivos intermediários apresentados no local apropriado? & 6,0 \\
\hline & G4 & Objetivos intermediários apresentados claramente? & 6,0 \\
\hline
\end{tabular}




\begin{tabular}{|c|c|c|c|}
\hline & G5 & Eu entendo os objetivos do aprendizado através do jogo? & 7,0 \\
\hline & \multicolumn{2}{|r|}{ Média da dimensão } & 6,4 \\
\hline \multirow{7}{*}{ Feedback } & F1 & Recebo feedback do meu progresso no jogo? & 6,0 \\
\hline & F2 & Recebo feedback imediato das minhas ações? & 6,5 \\
\hline & F3 & Sou notificado sobre novas tarefas imediatamente? & 7,0 \\
\hline & F4 & Sou notificado sobre novos eventos imediatamente? & 7,0 \\
\hline & F5 & $\begin{array}{l}\begin{array}{l}\text { Recebo informação sobre sucesso ou falha de objetivos } \\
\text { intermediários imediatamente? }\end{array} \\
\end{array}$ & 7,0 \\
\hline & F6 & Recebo informação sobre o meu status, como nível ou pontuação? & 7,0 \\
\hline & \multicolumn{2}{|r|}{ Média da dimensão } & 6,8 \\
\hline \multirow{8}{*}{ Imersão } & I1 & Esqueço do tempo enquanto jogo? & 4,5 \\
\hline & $\mathrm{I} 2$ & Esqueço das coisas ao meu redor enquanto jogo? & 5,0 \\
\hline & $\mathrm{I} 3$ & Esqueço dos problemas do dia-a-dia enquanto jogo? & 6,0 \\
\hline & I4 & Sinto uma noção de tempo alterada? & 4,5 \\
\hline & I5 & Posso ficar envolvido com o jogo? & 5,5 \\
\hline & I6 & Me sinto emocionalmente envolvido com o jogo? & 3,5 \\
\hline & $\mathrm{I} 7$ & Me sinto visceralmente envolvido com o jogo? & 3,5 \\
\hline & \multicolumn{2}{|r|}{ Média da dimensão } & 4,6 \\
\hline \multirow{7}{*}{$\begin{array}{l}\text { Interação } \\
\text { Social }\end{array}$} & S1 & Me sinto cooperativo com outros colegas? & 4,0 \\
\hline & $\mathrm{S} 2$ & Colaboro muito com outros colegas? & 4,0 \\
\hline & S3 & Cooperação no jogo auxilia no aprendizado? & 4,5 \\
\hline & S4 & O jogo suporta interação social (chats, etc...)? & 1,0 \\
\hline & S5 & O jogo suporta comunidades dentro do jogo? & 1,5 \\
\hline & S6 & O jogo suporta comunidades fora do jogo? & 2,0 \\
\hline & \multicolumn{2}{|r|}{ Média da dimensão } & 2,8 \\
\hline \multirow{6}{*}{$\begin{array}{l}\text { Melhoria do } \\
\text { Conhecimento }\end{array}$} & $\mathrm{K} 1$ & O jogo melhora meu conhecimento? & 7,0 \\
\hline & $\mathrm{K} 2$ & Capto as ideias básicas do conteúdo apresentado? & 7,0 \\
\hline & $\mathrm{K} 3$ & Tento aplicar o conhecimento no jogo? & 6,0 \\
\hline & K4 & O jogo motiva o jogador a integrar o conteúdo apresentado? & 6,5 \\
\hline & $\mathrm{K} 5$ & Quero saber mais sobre o conteúdo apresentado? & 7,0 \\
\hline & \multicolumn{2}{|r|}{ Média da dimensão } & 6,7 \\
\hline
\end{tabular}

Com base na escala Likert supracitada, médias superiores a quatro para cada dimensão atestam que o jogo atendeu à maioria dos seus itens. Conforme ilustrado pela Tabela 1, RIPChallenge obteve médias muito boas na maioria das dimensões. A seguir, será realizada uma análise das avaliação em cada uma das dimensões.

A média obtida na dimensão concentração $(6,1)$ atesta que RIPChallenge é, em termos gerais, um jogo que prende a atenção, oferece uma carga de trabalho adequada e tem suas atividades voltadas à aprendizagem. A média obtida na dimensão desafios $(5,3)$ é um relativamente mais baixa principalmente porque o jogo não conta com suporte online ou vídeos/áudios explicativos. Contudo, a avaliação nesta dimensão conclui que o jogo oferece tanto desafios adaptáveis a jogadores de habilidades variadas quanto a progressão daqueles desafios num ritmo condizente com a evolução das habilidades dos jogadores. Isto se dá pela presença dos dois modos de jogo descritos na Seção 5 .

A despeito da média na dimensão autonomia ser relativamente alta $(5,7)$, atestando que o jogadores possuem uma sensação geral de controle sobre o jogo, percebe-se pela menor nota atribuída nesta dimensão que eles não possuem a possibilidade de utilizar estratégias diferentes. Trata-se de algo já esperado, haja vista que há apenas uma estratégia para fazer com que a maior quantidade possível de pacotes seja roteada: clicar nos roteadores assim que possível para que eles dispararem suas atualizações. Aliás, o fato de o jogo deixar claro desde o início que seu objetivo é permitir que a maior quantidade possível de pacotes seja roteada explica a média elevada obtida na dimensão clareza dos objetivos $(6,4)$. 
A média obtida na dimensão feedback $(6,8)$ é explicada pelo fato de o jogo notificar adequadamente os jogadores acerca de seu progresso no jogo e da corretude de suas ações. As notificações são fornecidas de forma visual pelo placar, pelas escalas de progresso de nível e pelas imagem dos pacotes roteados/descartados, e de forma auditiva pelo som dos pacotes roteados/descartados.

Com relação à dimensão imersão, a média relativamente baixa $(4,6)$ pode ser explicada parcialmente pelo gênero do jogo. Apesar de contar com uma trilha sonora e sons importados de assets bem avaliados, RIPChallenge não se trata de um jogo de aventura ou RPG, gêneros que são mais propensos a proporcionar imersão aos seus jogadores. Porém, a média mais baixa de todas é a da dimensão interação social $(2,8)$, e isto é explicado pelo fato do jogo ter sido projetado para ser jogado de forma individual e assíncrona, não requerendo interações sociais. Neste contexto, seu projeto é diametralmente oposto aos jogos correlatos descritos na Seção 4.

A média elevada obtida na dimensão melhoria do conhecimento $(6,7)$ se deve ao fato de o jogo exibir, à exaustão, a dinâmica das atualizações de rota e a sua relação com as entradas nas tabelas de rotas. Estes são os pontos de maior confusão por parte dos alunos no aprendizado do RIP , e o jogo de fato contribui para a sua compreensão e memorização.

\section{Conclusões}

Este artigo apresentou RIPChallenge, um jogo sério para auxílio ao aprendizado do protocolo RIP. Naturalmente, o jogo não provê cenários tão realistas quanto as ferramentas descritas na Seção 3. Contudo, se forem tomados como base os trabalhos descritos na Seção 4 pode-se afirmar que o nível de abstração utilizado no jogo é mais baixo do que o utilizado pelo jogo de labirinto apresentado em [Graven et al 2009]; porém não tão baixo quanto em [Arevalillo-Herráez et al 2012], que descreve um jogo no qual as interfaces de rede de um roteador têm que ser configuradas pelo jogador.

De fato, o foco de RIPChallenge foi estabelecido na dinâmica das atualizações e tabelas de rotas - e não na representação real daqueles elementos - porque aqueles primeiros são os tópicos que apresentam a maior dificuldade de assimilação por parte dos alunos. Com relação à disponibilidade de uso, o fato de o seu desenvolvimento ser realizado com o auxílio do motor de jogos Unity faz com que o jogo possa ser utilizado em várias plataformas. Além disso não são necessárias várias pessoas para jogá-lo, como em todos os trabalhos relacionais - especialmente em [Lemcke et al 2015].

Naturalmente, o fato do jogo ser jogado por apenas um jogador cobra o preço de não haver interações sociais diretas entre jogadores. Interações sociais indiretas via Web poderiam acontecer a partir da criação de comunidades virtuais sobre o jogo onde informações de desempenho dos jogadores seriam compartilhadas; todavia, a implantação do gameplay envolvendo vários jogadores passa por um reprojeto do jogo.

A despeito do protótipo ter sido avaliado positivamente, algumas sugestões foram dadas no sentido de melhorar a sua funcionalidade. Dentre elas, está a presença de fases que contemplem topologias evoluídas a partir da atual. Mais precisamente, uma nova fase incluiria um enlace entre R1 e R3 para cobrir situações em que um determinado roteador recebe duas rotas para uma mesma rede e escolhe a de menor custo. Outra fase incluiria um roteador adicional formando uma topologia na qual há dois caminhos com mesmo custo até uma determinada rede. Melhorias estéticas são também previstas. $\mathrm{O}$ protótipo pode ser obtido a partir de https://ripchallenge. wixsite. com/index. 


\section{Referências}

Arevalillo-Herráez, M., Morán-Gómez, R. e Claver, J. M. (2012) "Conquer the Net: An Educational Computer Game to Learn the Basic Configuration of Networking Components", Computer Applications in Education Engineering, Volume: 20, edição: 1, páginas 72-77. Doi:10.1002/cae.20374;

Cisco Systems (2019) "Introduction to Packet Tracer: Discover and troubleshoot using powerful networking simulation tool". Disponível em https://www.netacad.com/courses/packet-tracer/introduction-packet-tracer;

Csikszentmihalyi, M. (1990) "Flow: The Psychology of Optimal Experience", Harper \& Row.

Fu, F., Su, R. e Yu, S. (2009) "EGameFlow: A scale to measure learners' enjoyment of e-learning games", Computers \& Education, Volume: 52, edição: 1, páginas 101-112. Doi:10.1016/j.compedu.2008.07.004;

GNS3 Technologies Inc. (2019) "GNS3: The software that empowers network professionals". Disponível em https://www.gns3.com/;

Graven, O. H., Hansen, H. A. e MacKinnon, L. (2009) “A Computer Game Modelling Routing in Computer Networks as Abstract Learning Material in a Blended Learning Environment", International Journal of Emerging Technologies in Learning (iJET), Volume 4, páginas 18-22. Doi:10.3991/ijet.v4s3.1081;

Hays, R. (2005) "The Effectiveness of Instructional Games: a Literature Review and Discussion". Technical Report 2005-004. Naval Air Warfare Center Training System Division. Disponível em http://www.dtic.mil/dtic/tr/fulltext/u2/a441935.pdf;

Hedrick, C. (1988) "Routing Information Protocol”, RFC 1058. Disponível em https://tools.ietf.org/html/rfc1058;

Kapp, K. (2012) "The Gamification of Learning and Instruction: Game-Based Methods and Strategies for Training and Education", Pfeiffer \& Company, $1^{\text {a }}$ Edição;

Kurose, J. e Ross, K. (2013) “ Redes de Computadores e a Internet: Uma Abordagem Top-Down”, Pearson Universidades, 6 a Edição;

Lemcke, S., Haedge, K.. Zender, R. e Lucke, U. (2015) "RouteMe: a Multilevel Pervasive Game on Mobile Ad hoc Routing", Personal and Ubiquitous Computing, Volume: 19, edição: 3-4, páginas 537-549. Doi:10.1007/s00779-015-0843-2;

Malkin, G. (1998) "RIP Version 2", RFC 2453. Disponível em https://tools.ietf.org/html/rfc2453;

Michael, D. e Chen, S. (2005) "Serious Games: Games That Educate, Train, and Inform", Course Technology PTR;

Sweetser, P. e Wyeth, P. (2006) "GameFlow: A Model for Evaluating Player Enjoyment in Games ", ACM Computers in Entertainment 3-3, páginas 1-24. Doi: 10.1145/1077246.1077253;

Tanenbaum, A. S. e Wetherall, D. (2011) "Redes de Computadores", Pearson Universidades, $5^{\text {a }}$ Edição;

Vogel, Jennifer J., Vogel, David S., Cannon-Bowers, Jan, Bowers, Clint A., Muse, Kathryn e Wright, Michelle (2006) "Computer Gaming and Interactive Simulations for Learning: A Meta-Analysis". Journal of Educational Computing Research, Volume: 34, edição: 3, páginas: 229-243; 